\title{
The anticancer mechanism of 2'-hydroxycinnamaldehyde in human head and neck cancer cells
}

\author{
SANG-GUN AHN ${ }^{1}$, YOUNG-HEE JIN ${ }^{2}$, JUNG-HOON YOON ${ }^{3}$ and SOO-A KIM ${ }^{2}$ \\ ${ }^{1}$ Department of Pathology, School of Dentistry, Chosun University, Gwangju 501-759; \\ ${ }^{2}$ Department of Biochemistry, College of Oriental Medicine, Dongguk University, Gyeongju 780-714; \\ ${ }^{3}$ Department of Oral and Maxillofacial Pathology, College of Dentistry, Daejeon Dental Hospital, Wonkwang \\ Bone Regeneration Research Institute, Wonkwang University, Daejeon 302-120, Republic of Korea
}

Received June 18, 2015; Accepted August 13, 2015

DOI: $10.3892 /$ ijo.2015.3152

\begin{abstract}
Cinnamaldehyde has been shown to effectively induce apoptosis in a number of human cancer cells. In the present study, cinnamaldehyde derivative-induced apoptosis and its signaling pathways were assessed in p53-wild (SGT) and p53-mutant (YD-10B) human head and neck cancer cells. The cinnamaldehyde derivatives, 2'-hydroxycinnamaldehyde (HCA) and 2'-benzoyloxycinnamaldehyde (BCA), exhibited powerful anti-proliferative effects on SGT and YD-10B cells. The apoptotic effect induced by HCA or BCA was supported by caspase-3, -7, -9 and PARP activation, and confirmed by Annexin V-FITC/PI double staining. HCA induced the expression of p21 in both SGT and YD-10B cells. Furthermore, HCA induced the level of pro-apoptotic Bak1 expression while decreasing the level of anti-apoptotic $\mathrm{Bcl}-2$ in both cell lines, suggesting that HCA induced the cell death pathway in a p53-independent manner. HCA also induced the expression of LC3B in SGT and YD-10B cells. Following pre-incubation with the autophagy inhibitor 3-MA, HCA-induced apoptosis was largely increased in SGT cells, while inhibited in YD-10B cells, suggesting that autophagy may actively contribute to HCA-induced apoptosis. Taken together, these observations suggest that HCA may be an effective therapeutic agent in the treatment of head and neck cancer regardless of p53 status.
\end{abstract}

\section{Introduction}

Cinnamaldehyde exhibits various cellular effects, including antitumor, anti-angiogenesis and anti-inflammatory

Correspondence to: Dr Soo-A Kim, Department of Biochemistry, College of Oriental Medicine, Dongguk University, 123 Dongdae-ro, Gyeongju, Gyeongsangbuk-do 780-714, Republic of Korea E-mail:ksooa@dongguk.ac.kr

Key words: cinnamaldehyde, p53, apoptosis, autophagy, head and neck cancer cells activities (1-3). Several studies have shown that the cinnamaldehyde derivatives, 2'-hydroxycinnamaldehyde (HCA) and 2'-benzoyloxycinnamaldehyde (BCA), exhibit antitumor activities by inducing cell cycle arrest and the production of reactive oxygen species (ROS) in a variety of human cancer cells, including breast, leukemia, ovarian, lung and colon cancer (4-8). In addition, HCA causes ER stress through the inhibition of the proteasome pathway and mitochondrial perturbation, which are associated with the induction of apoptosis in SW620 colon cancer cells (9). Our previous study demonstrated the potential effects of HCA and BCA on SCC-15 and Hep-2 human oral cancer cells (10). BCA is a well-known derivative of HCA that induces the expression and nuclear translocation of EGR1 and the expression of its target genes, including activating transcription factor 3 (ATF3), NSAID-activated gene 1 protein (NAG-1), and growth arrest and DNA-damage-inducible protein $\alpha$ (GADD45A) in prostate cancer cells (11). BCA has shown therapeutic selectivity in a K-ras-transformed animal model through the downregulation of antioxidants (12). We have also shown that BCA mainly exerts its anti-inflammatory effects through the inhibition of the JNK pathway, thereby leading to AP-1 transcriptional activity (13). However, little is known about the mechanistic relationship between autophagy and cinnamaldehyde derivative-induced apoptosis.

Oral squamous cell carcinoma (OSCC) is an aggressive disease that is histologically characterized as hyperplasia, dysplasia, carcinoma in situ and invasive oral cancers $(14,15)$. Patients with premalignant oral lesions have an increased risk of developing OSCC and molecular processes, such as tumor suppressor gene inactivation or oncogene activation, leading to progression of precancerous lesions to invasive oral cancers $(14,16)$. Finding a novel pharmacologic agent that can effectively halt the process of oral carcinogenesis is one of successful oral cancer chemoprevention tools and a variety of agents are being tested to prevent progression to invasive cancer $(14,16)$.

In the present study, we demonstrated that HCA- or BCA-induced apoptosis was independent of p53 status in p53-wild-type SGT cells and p53-mutant YD-10B cells. We also investigated the roles of autophagy in cinnamaldehyde derivative-induced apoptosis in human head and neck cancer 
cells and showed autophagy participates in the regulation of cell death by enhancing apoptosis. These findings provide new information on the relationship between autophagy and apoptosis in HCA treated p53-wild-type and p53-mutant oral cancer cells.

\section{Materials and methods}

Cell culture and reagents. The salivary gland adenocarcinoma cell line (SGT, p53-wild-type) and oral squamous cell carcinoma cell line (YD-10B, p53-mutant) were maintained in Dulbecco's modified Eagle's medium (DMEM) or RPMI-1640 medium, respectively. The YD-10B cell line contains a point mutation in p53 at codon 236 of exon 7, resulting in the change of codon 236 from TAC to TAA (17). Both cell lines were cultured in medium supplemented with $10 \%$ fetal bovine serum (FBS), 100 units $/ \mathrm{ml}$ of penicillin, and $100 \mu \mathrm{g} / \mathrm{ml}$ of streptomycin and were maintained at $37^{\circ} \mathrm{C}$ in a humidified incubator with a $5 \% \mathrm{CO}_{2}$ atmosphere. 2'-Hydroxycinnamaldehyde (HCA) and 2'-methoxycinnamaldehyde (MCA) were purchased from Santa Cruz Biotechnology (Santa Cruz, CA, USA). 2'-Benzoyloxycinnamaldehyde (BCA) and 2'-acetoxycinnamaldehyde (ACA) were a generous gift of Dr S.H. Hong (Department of Microbiology, School of Dentistry, Kyungpook National University, Daegu, Korea).

Cell proliferation assay. The cells were seeded in 12-well plates at a density of $5 \times 10^{5}$ cells $/ \mathrm{ml}$. The cells were then cultured overnight and were treated with various concentrations of HCA or BCA for $24 \mathrm{~h}$. The cell viability was measured using the MTT assay according to the previously described method (18). In brief, $24 \mathrm{~h}$ after treatment, the cells were washed twice with ice-cold PBS, and $0.25 \mathrm{ml}$ of cell culture medium and $25 \mu 1$ of 3-(4,5-dimethylthiazol-2-yl)2,5-diphenyltetrazolium bromide solution $(5 \mathrm{mg} / \mathrm{ml}$ in PBS) was added. After $3 \mathrm{~h}$ of incubation, the medium was removed and $125 \mu \mathrm{l}$ of acidisopropanol $(0.04 \mathrm{~mol} / 1 \mathrm{HCl}$ in isopropanol) was added. The absorbance was measured at a wavelength of $570 \mathrm{~nm}$, and the results were plotted as the means \pm SD of three separate experiments.

Cell cytotoxicity assay. Cell cytotoxicity was evaluated by measuring the activity of lactate dehydrogenase (LDH) after treatment with HCA or BCA using a CytoTox $96^{\circledR}$ Non-Radioactive Cytotoxicity Assay kit (Promega, Madison, WI, USA) according to the manufacturer's instruction. The optical density was assessed at a wavelength of $490 \mathrm{~nm}$.

Western blotting. Cells were treated with various concentrations of HCA or BCA for the indicated time periods. The cells were then washed with PBS and harvested in lysis buffer. Samples containing equal amounts of protein were loaded into each lane of an SDS-polyacrylamide gel for electrophoresis and were subsequently transferred onto a PVDF membrane. The membranes were blocked and then incubated with antibodies. Antibodies against Bak1 (\#3814), Bcl-2 (\#2876), Bid (\#2002), LC3B (\#3868), caspase-7 (\#9492) and p-p53 (\#9284) were purchased from Cell Signaling Technology (Beverly, MA, USA). The p53 (sc-126), caspase-3 (sc-7148), caspase-9 (sc-7885) and PARP (sc-7150) antibodies were from Santa
Cruz Biotechnology and antibodies against $\beta$-actin (A1978) were purchased from Sigma-Aldrich (St. Louis, MO, USA).

Cell death evaluation. Apoptosis was measured using a cell death detection ELISA kit (Roche Molecular Biochemicals, Indianapolis, IN, USA). The relative apoptosis, which correlates with absorption at $405 \mathrm{~nm}$ with a reference wavelength of $490 \mathrm{~nm}$, was measured according to the manufacturer's instructions.

Annexin V-FITC/PI double staining. For the cell cycle analysis, cells were harvested and fixed with $70 \%$ ethanol for $1 \mathrm{~h}$ at $4^{\circ} \mathrm{C}$. After washing with cold PBS, the cells were incubated with DNase-free RNase for $30 \mathrm{~min}$ at $37^{\circ} \mathrm{C}$. The specific binding of Annexin V-FITC/PI was performed by incubating the cells for $15 \mathrm{~min}$ at room temperature in a binding buffer (10 mM HEPES, pH 7.4, $140 \mathrm{mM} \mathrm{NaCl,} 2.5 \mathrm{mM} \mathrm{CaCl}$ ) containing saturated concentrations of Annexin V-FITC and PI. Apoptotic cells were visualized with a Nikon Eclipse E800 automated fluorescence microscope (Nikon, Tokyo, Japan). For the flow cytometric analysis, the cells were measured with a FACSCalibur flow cytometer (BD Biosciences, San Jose, CA, USA) using CellQuest software.

Quantification of acidic vesicular organelles. Autophagy was characterized by the formation of acidic vesicular organelles (AVOs). Cells were seeded in $6-\mathrm{cm}^{2}$ plates at a density of $5 \times 10^{5}$ cells $/ \mathrm{ml}$. After treatment with HCA for $24 \mathrm{~h}$, acridine orange $(1 \mu \mathrm{g} / \mathrm{ml})$ was added to the living cells for $30 \mathrm{~min}$, and the cells were removed from the plate with trypsin-EDTA and collected in phenol red-free growth medium. Approximately $1 \times 10^{4}$ cells were illuminated with blue $(488 \mathrm{~nm})$ excitation light, and the green $(510-530 \mathrm{~nm})$ and red $(650 \mathrm{~nm})$ fluorescent emissions were measured using a flow cytometer (Beckman Coulter, Inc., Brea, CA, USA).

MDC staining. To observe autophagy formation, cells were grown on glass coverslips for $24 \mathrm{~h}$. After treatment with HCA for $24 \mathrm{~h}$, the cells were treated with $0.05 \mathrm{mM}$ monodansylcadaverine (MDC; Sigma-Aldrich) at $37^{\circ} \mathrm{C}$ in $5 \% \mathrm{CO}_{2}$ for $10 \mathrm{~min}$. The cells were then fixed with $4 \%$ paraformaldehyde in PBS for $10 \mathrm{~min}$. Following incubation, the cells were washed three times with PBS and were immediately analyzed under a fluorescence microscope (IX-71; Olympus, Tokyo, Japan). The fluorescence was measured at an excitation wavelength of $380 \mathrm{~nm}$ with an emission filter at $530 \mathrm{~nm}$.

siRNA experiment. The siRNA construct for Atg5 was obtained in the form of select validated siRNA (Bioneer Corp., Daejeon, Korea). The cells were transfected with 20 nM siRNA using the Lipofectamine RNAi Max Transfection reagent (Invitrogen, Carlsbad, CA, USA) according to the manufacturer's instructions. The cells were harvested $24 \mathrm{~h}$ after the transfection. The total cell lysates were separated by SDS-PAGE and were analyzed by western blot analysis, as described above.

Statistical analysis. The statistical analysis was performed with data obtained from three independent experiments. The data are represented as the means \pm SD. The ANOVA 

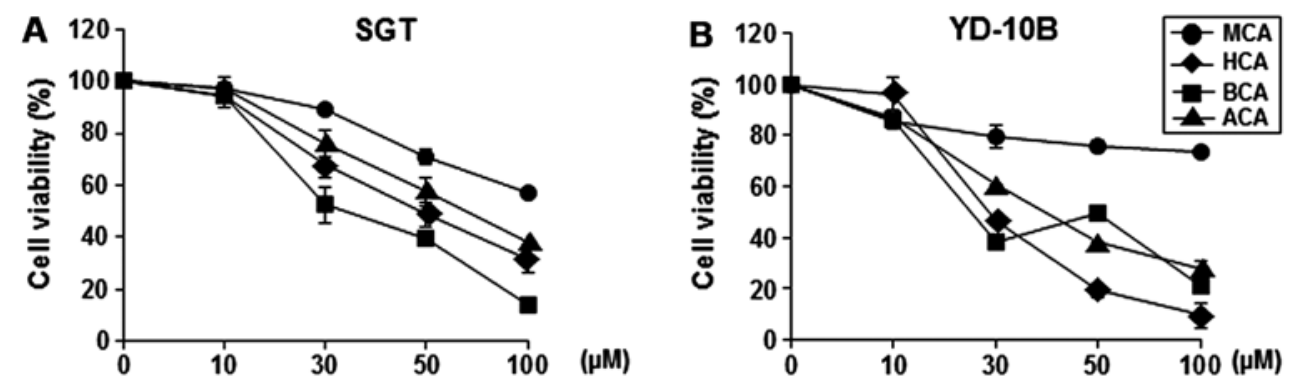

Figure 1. Anti-proliferative effects of cinnamaldehyde derivatives on human head and neck cancer cells (HNCC). (A) SGT and (B) YD-10B cells were treated with the indicated concentrations of cinnamaldehyde derivatives for $24 \mathrm{~h}$. The cell proliferation was estimated using the MTT assay. The data are expressed as the means \pm SD of the results from three separate experiments.
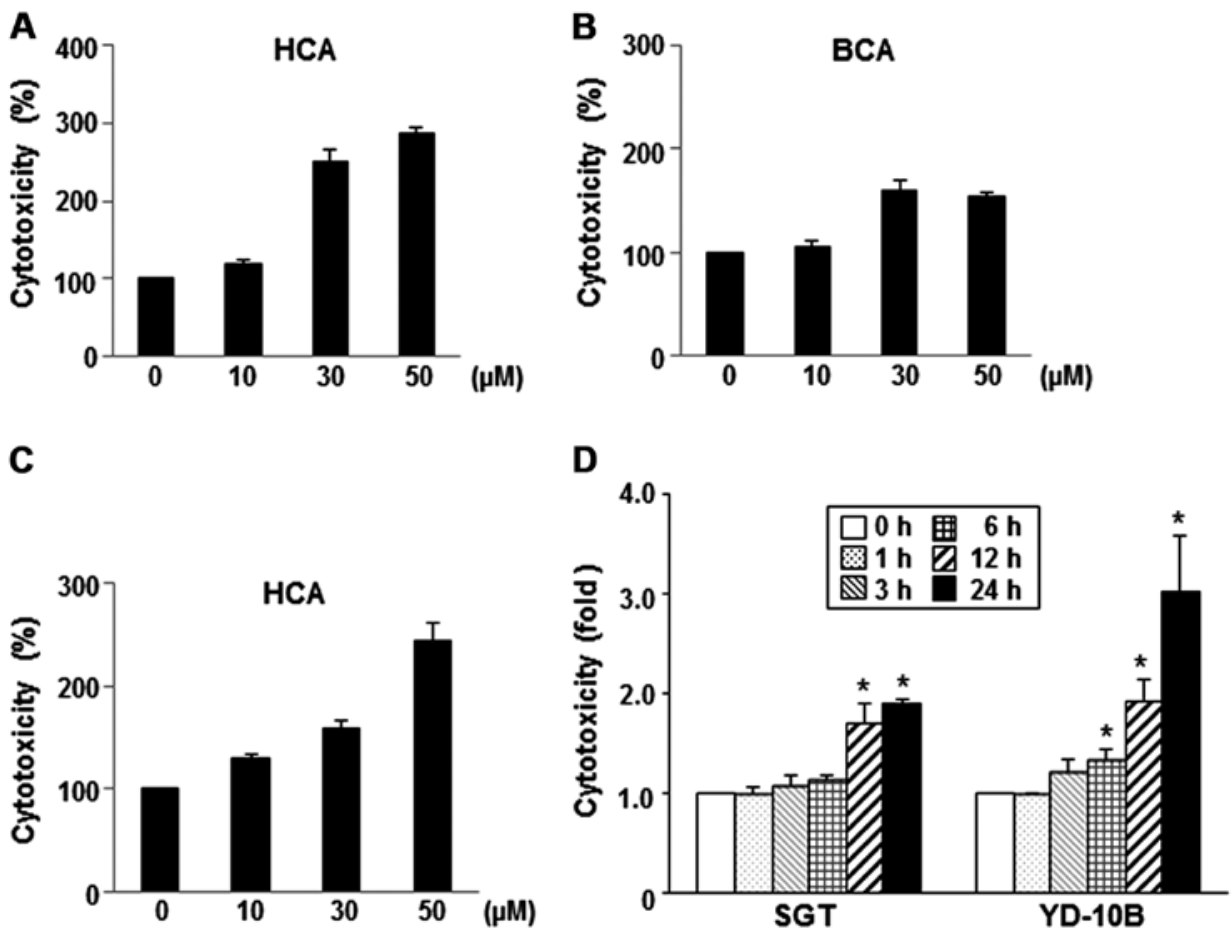

Figure 2. The cytotoxic effects of HCA and BCA in SGT and YD-10B cells. (A and B) SGT and (C) YD-10B cells were treated with the indicated concentrations of HCA or BCA for $24 \mathrm{~h}$. (D) The cells were treated with $50 \mu \mathrm{M} \mathrm{HCA}$ for the indicated time periods. Cell cytotoxicity was measured using the LDH assay. The data were expressed as the means $\pm \mathrm{SD}$ of the results from three separate experiments. ${ }^{*} \mathrm{P}<0.05$ compared with HCA-untreated control cells.

and Student's t-test were applied to determine the statistical significance. P-values $<0.05$ were considered to be significant.

\section{Results}

Effect of cinnamaldehyde derivatives on cell proliferation and cytotoxicity in p53-wild-type SGT and p53-mutant $Y D-10 B$ cells. We investigated the cinnamaldehyde derivatives (MCA, HCA, BCA and ACA) to develop a potential antitumor agent. To assess the growth-inhibitory effect of these cinnamaldehyde derivatives, we initially examined their effects on the proliferation of the SGT and YD-10B cells. As shown in Fig. 1, HCA and BCA showed more potent growth-inhibitory effects than the other tested cinnamaldehyde derivatives (MCA and ACA) against SGT and YD-10B cells, with $\mathrm{IC}_{50}$ values ranging from 30 to $50 \mu \mathrm{M}$. In particular, HCA showed the most potent growth-inhibitory effect in YD-10B cells compared to all other cinnamalde- hyde derivatives. Treatment with $50 \mu \mathrm{M}$ of HCA reduced the cell viability by $\sim 81 \%$ (Fig. 1B).

To evaluate the dose-dependent cytotoxic effects of HCA and $\mathrm{BCA}$, the cells were incubated with varying concentrations of HCA or BCA for $24 \mathrm{~h}$. As shown in Fig. 2A and B, HCA and BCA caused dose-dependent cell cytotoxicity compared to the untreated SGT cells. When the YD-10B cells were treated with HCA for $24 \mathrm{~h}$, the cell cytotoxicity also increased in a dosedependent manner (Fig. 2C). A constant concentration of HCA $(50 \mu \mathrm{M})$ was applied to SGT and YD-10B cells for different time periods, and the cytotoxicity was measured relative to untreated control cells. Fig. 2D showed that HCA induced high cytotoxicity between 12 and $24 \mathrm{~h}$ in both SGT and YD-10B cells. Similar effects were noted in the BCA-treated cells (data not shown).

HCA treatment leads to the upregulation of p53-independent p21 and Bakl. It has been reported that p53 modulates the 
A

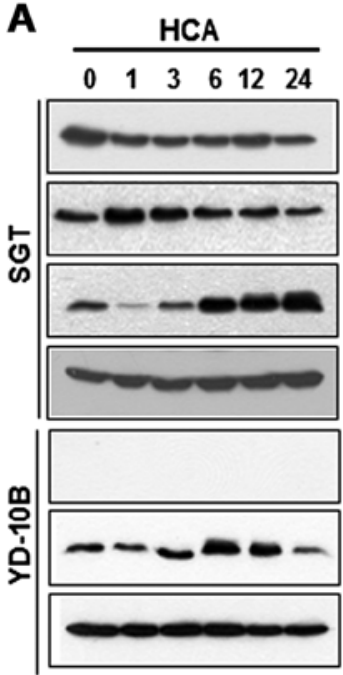

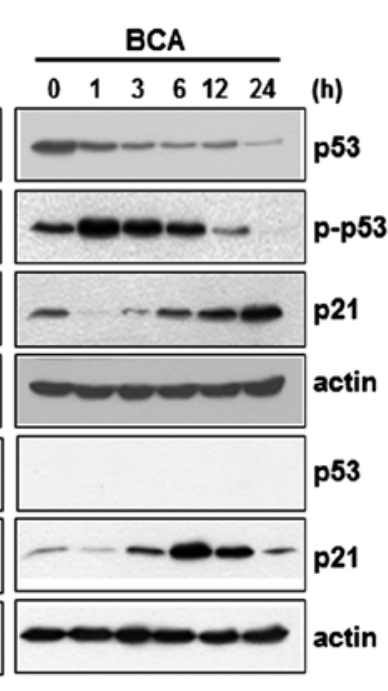

B

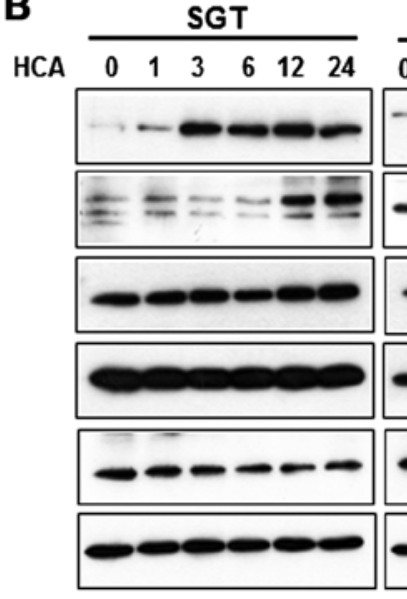

YD-10B
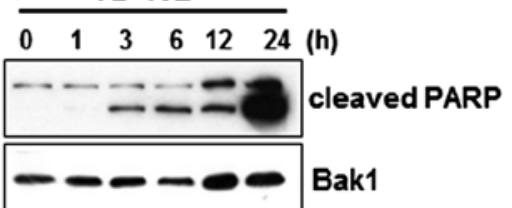

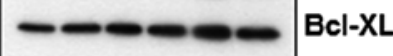

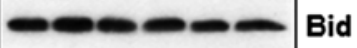

$-\infty-\infty \mathrm{Bcl}-2$

Figure 3. HCA and BCA induce the expression of p21 and Bak1. SGT and YD-10B cells were treated with $50 \mu \mathrm{M}$ HCA or BCA for the indicated time periods (A) Western blot analysis was performed to detect the expression levels of p53, p-p53 and p21 proteins. (B) The expression levels of cleaved PARP, Bak1, Bcl-XL, Bid and Bcl-2 were detected by western blot analysis. Actin was used as a loading control.

A
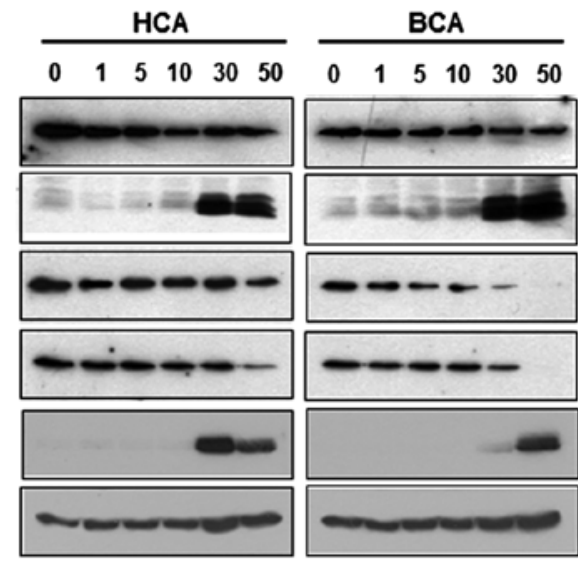

(

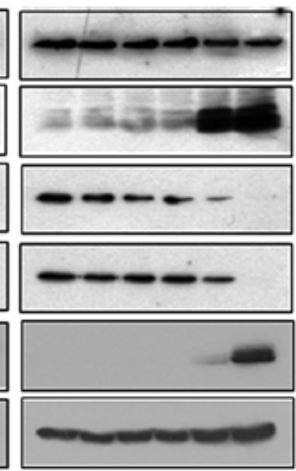

procaspase 3

caspase 3

procaspase 7

procaspase 9

cleaved PARP

actin
B

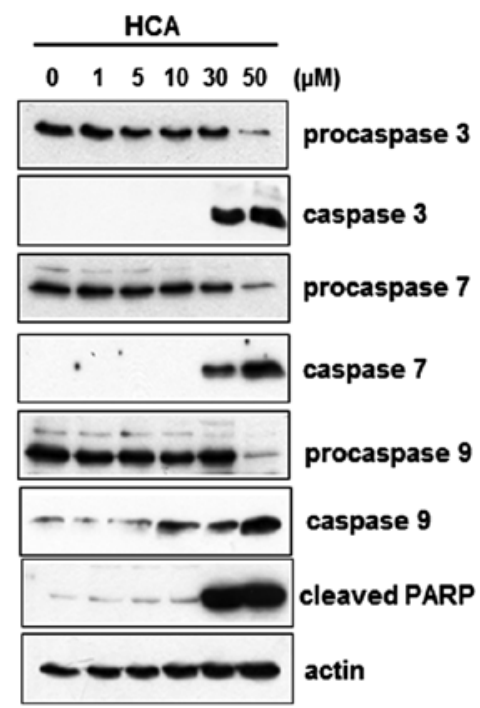

D

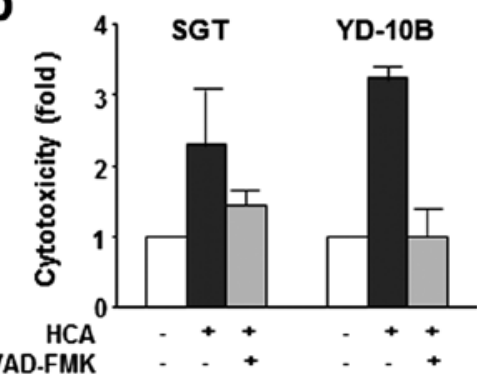

Figure 4. HCA and BCA induce apoptosis in SGT and YD-10B cells. (A) SGT and (B) YD-10B cells were treated with the indicated concentrations of HCA or BCA for $24 \mathrm{~h}$. Total cell lysates were prepared and the expression levels of procaspase-9, caspase-9, procaspase-7, caspase-7, procaspase-3, caspase-3 and cleaved PARP were detected by western blot analysis. (C) SGT and YD-10B cells were pre-treated with $30 \mu \mathrm{M} \mathrm{z}$-VAD-FMK for $16 \mathrm{~h}$ and then incubated with $50 \mu \mathrm{M}$ HCA for an additional $24 \mathrm{~h}$. The levels of PARP and cleaved PARP were then monitored by western blot analysis. Actin was used as a loading control. (D) The cells were incubated in the presence or absence of z-VAD-FMK for $16 \mathrm{~h}$ and were then treated with HCA for $24 \mathrm{~h}$. The cell cytotoxicity was measured using the $\mathrm{LDH}$ assay. The data were expressed as the means $\pm \mathrm{SD}$ of three separate experiments.

activation and oligomerization of Bax/Bak, which leads to mitochondria-mediated cell death $(19,20)$. To examine the importance of p53 in HCA- or BCA-induced cell growth inhibition, we performed western blot analysis using anti-p53 and 
A

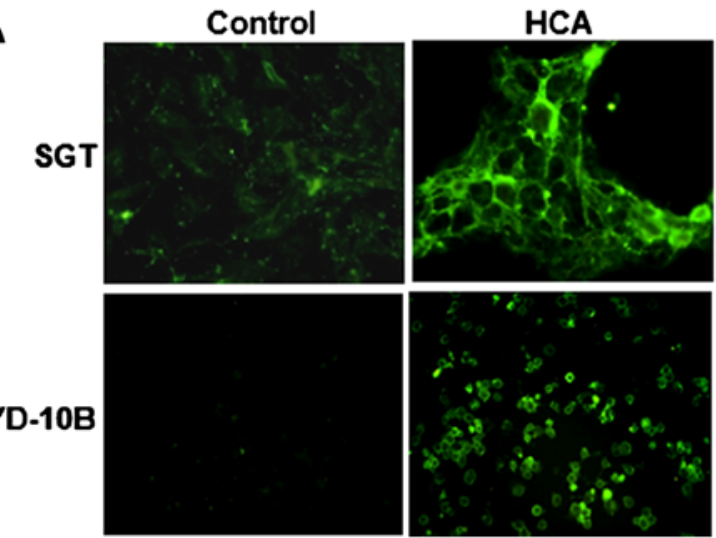

B
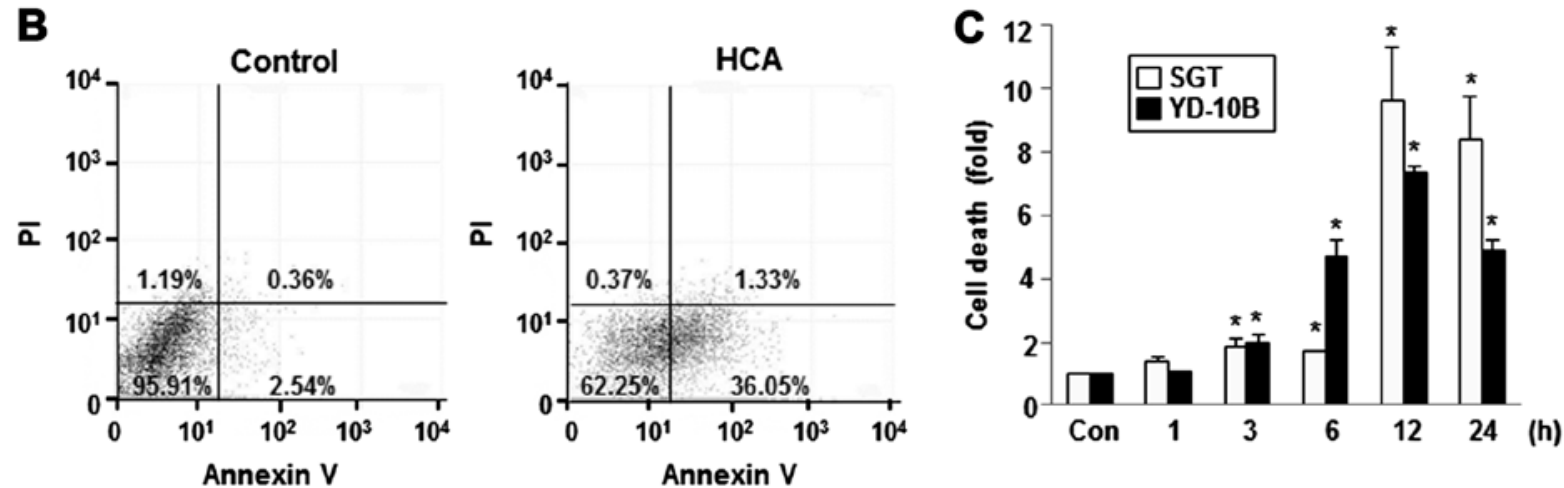

Figure 5. Evaluation of apoptosis in HCA-treated SGT and YD-10B cells. (A) The cells were treated with $50 \mu \mathrm{M}$ HCA for $24 \mathrm{~h}$ and then stained with Annexin V-FITC/PI. Apoptosis was determined by fluorescence microscopy. (B) YD-10B cells were treated with $50 \mu \mathrm{M}$ HCA for $24 \mathrm{~h}$ and then stained with Annexin V-FITC/PI. Apoptosis was detected by flow cytometry. (C) SGT and YD-10B cells were treated with $50 \mu \mathrm{M}$ HCA for the indicated time periods. The relative apoptosis of cells was determined using a cell death detection ELISA kit as described in Materials and methods. The data are expressed as the means \pm SD of three individual experiments. ${ }^{*} \mathrm{P}<0.05$ compared with HCA-untreated control cells.

anti-p21 antibodies. Cells were treated with HCA or BCA for various time periods, and the cell lysates were prepared. As shown in Fig. 3A, HCA or BCA treatment caused significant p21 induction as well as p53 phosphorylation in SGT cells. p21 induction was also observed in YD-10B cells, whereas p53 phosphorylation was not detected. These results clearly showed that HCA and BCA can inhibit cell growth in YD-10B cells through the p53-independent induction of p21, suggesting that p53 does not play an essential role in cell growth inhibition in YD-10B cells.

Bcl-2 family proteins also play a critical role in the regulation of the mitochondria-dependent cell death pathway (21). To investigate whether HCA affects the levels of pro-apoptotic proteins (Bax and Bak1), BH3-only protein (Bid), and anti-apoptotic proteins (Bcl-2 and Bcl-XL), SGT and YD-10B cells were treated with HCA and western blot analysis was performed. We observed the time-dependent upregulation of Bak1 in SGT and YD-10B cells, while the level of anti-apoptotic protein $\mathrm{Bcl}-2$ was decreased in the cells treated with HCA (Fig. 3B).

HCA induces caspase-dependent apoptotic cell death. To address whether HCA induces apoptosis in SGT and YD-10B cells, both cell types were treated with HCA for $24 \mathrm{~h}$ and then assessed for caspase activity. As shown in Fig. 4A, HCA reduced the level of procaspase-3, -7 and -9 in SGT cells in a dose-dependent manner. In YD-10B cells, the protein levels of procaspase-3, -7 and -9 were also decreased by HCA treatment
(Fig. 4B). HCA largely increased the levels of cleaved PARP in both SGT and YD-10B cells (Fig. 4A and B).

To confirm the role of HCA in caspase-mediated apoptosis, SGT and YD-10B cells were treated with z-VAD-FMK (a pancaspase inhibitor) before exposure to HCA. Importantly, our data showed that z-VAD-FMK inhibited the HCA-induced PARP activation in both cell lines (Fig. 4C). Moreover, z-VADFMK substantially protected the cells against HCA-induced cytotoxicity (Fig. 4D). Collectively, these findings suggest that HCA induces the mitochondrial caspase-dependent apoptotic pathway in SGT and YD-10B cells.

To confirm these results, apoptotic cells were quantified using the Annexin V-FITC/PI double staining assay. After treatment with $50 \mu \mathrm{M} \mathrm{HCA}$ for $24 \mathrm{~h}$, apoptosis was observed by visualizing the green fluorescence in SGT and YD-10B cells using fluorescence microscopy. As shown in Fig. 5A, intense fluorescence was observed in the HCA-treated cells. After HCA treatment, a number of apoptotic cells were significantly increased in both cell lines. An increased number of Annexin V-positive cells (early apoptosis) was detected in the flow cytometric analysis after HCA treatment, indicating the onset of apoptosis in HCA-treated cells (Fig. 5B). In addition, a cell death detection ELISA clearly showed that treatment with $50 \mu \mathrm{M}$ HCA induced cell death in a time-dependent manner in both SGT and YD-10B cells (Fig. 5C).

HCA-induced autophagy regulates apoptosis. We next questioned whether HCA induces autophagy in SGT and YD-10B 
A

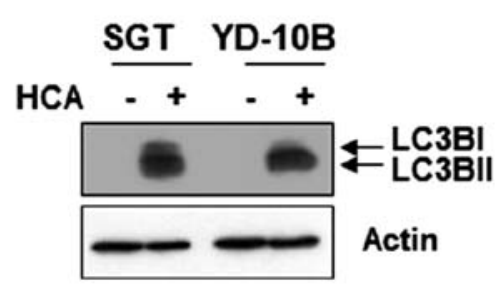

C

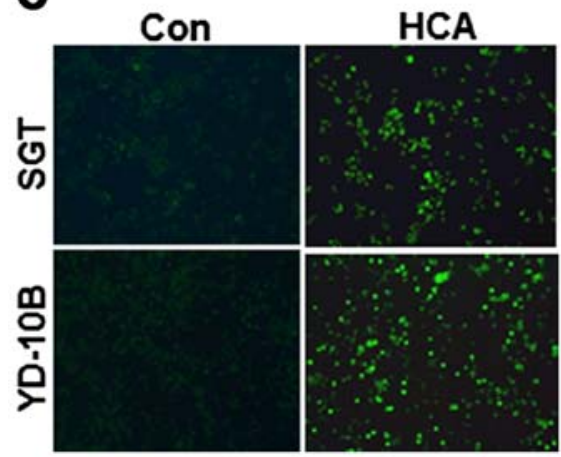

B

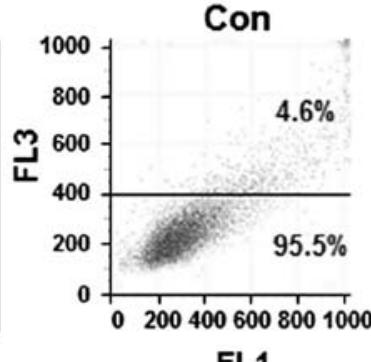

FL1

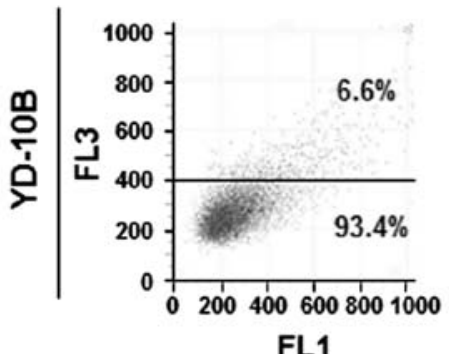

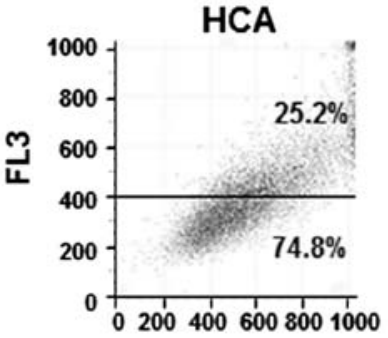

FL1

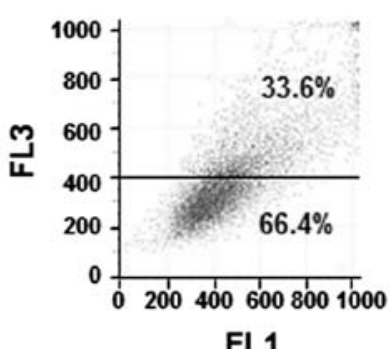

Figure 6. Induction of autophagy in HCA-treated cells. (A) The cells were treated with $50 \mu \mathrm{M}$ HCA for $24 \mathrm{~h}$ and the expression level of LC3B was determined by western blot analysis. (B) After treatment with HCA for $24 \mathrm{~h}$, cells were labeled with acridine orange $(1 \mu \mathrm{g} / \mathrm{ml})$ and autophagic cells were quantitated by flow cytometry. FL1 indicates green color intensity (cytoplasm and nucleus) and FL3 shows red color intensity (AVOs). (C) The cells were treated with HCA for $24 \mathrm{~h}$ and then stained with MDC for $10 \mathrm{~min}$. The cells were fixed and monitored by fluorescence microscopy.

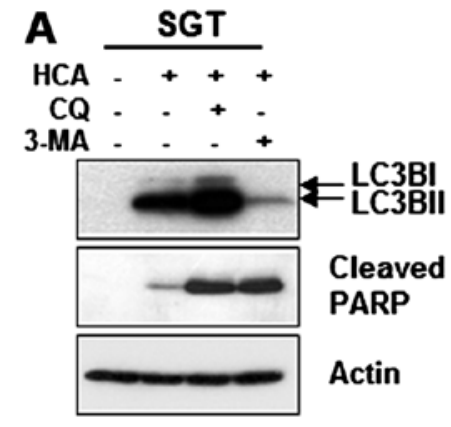

D

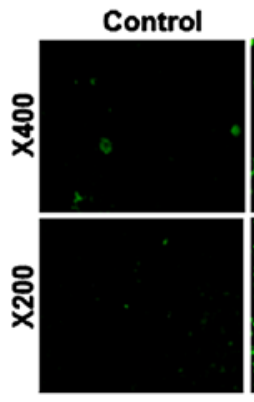

B
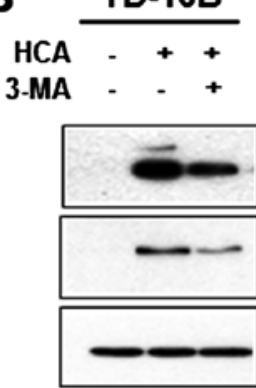

$\leftarrow$ LC3B $\div$ LC3Bil Cleaved PARP Actin
C

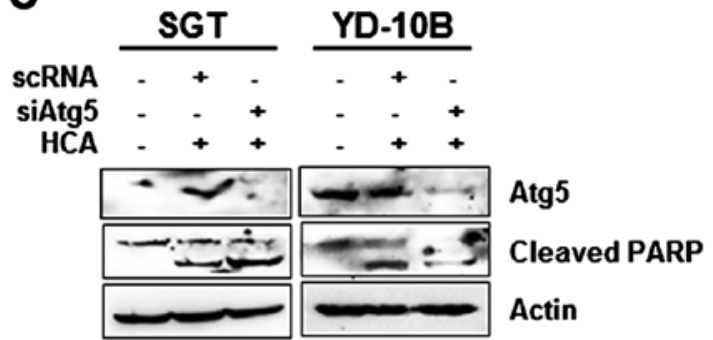

Figure 7. Autophagy modulates HCA-induced apoptosis. (A and B) The cells were pre-treated with 3-MA (2 mM) or CQ (50 $\mu \mathrm{M})$ for $1 \mathrm{~h}$ and then incubated with HCA $(50 \mu \mathrm{M})$ for $24 \mathrm{~h}$. The expression levels of LC3B and cleaved PARP were detected using western blot analysis. (C) The cells were transfected with either Atg5 siRNA or a non-silencing control siRNA for $24 \mathrm{~h}$ and then subsequently treated with HCA for $24 \mathrm{~h}$. The levels of Atg5 and cleaved PARP were measured by western blot analysis. (D) YD-10B cells were pre-treated with autophagy inhibitors for $1 \mathrm{~h}$ and were then incubated with HCA for $24 \mathrm{~h}$. The cells were stained with Annexin V-FITC/PI and observed by fluorescence microscopy. (E) The cells were transfected with plasmids expressing EGFP-LC3B or EGFP. The levels of EGFP, EGFP-LC3B, and cleaved PARP were detected by western blot analysis. Actin was used as a loading control.

cells. To address this issue, the cells were treated with $50 \mu \mathrm{M}$ HCA for $24 \mathrm{~h}$, and the level of LC3B, a marker for autophagy, was detected by western blot analysis. As shown in Fig. 6A, the levels of LC3B were increased in both HCA-treated cell lines.
To confirm the presence of autophagy, the HCA-treated cells were stained with acridine orange and the formation of characteristic acidic vesicular organelles (AVOs) was quantified by flow cytometry. As shown in Fig. 6B, the number of AVOs was increased in HCA-treated cells compare with untreated 
control cells. In the MDC staining assay, MDC-labeled vacuoles were weakly detected in the control cells. However, in the HCA-treated cells, the number of MDC-labeled cells was largely increased (Fig. 6C). Taken together, these results suggest that HCA induced autophagy in SGT and YD-10B cells.

We subsequently examined the relationship between apoptosis and autophagy in HCA-treated cells. To investigate potential cross-talk between HCA-induced autophagy and apoptosis, we examined whether HCA-induced apoptosis can be inhibited by an autophagy inhibitor. SGT and YD-10B cells were incubated with $50 \mu \mathrm{M}$ HCA for $24 \mathrm{~h}$ in the presence or absence of the autophagy inhibitors, chloroquine (CQ) or 3-methyladenine (3-MA), and apoptosis was evaluated by detecting the level of cleaved PARP. As shown in Fig. 7A, HCA induced PARP cleavage in SGT cells. Notably, the HCA-induced PARP activation strongly increased with the addition of CQ or 3-MA, even though the level of LC3B decreased in the 3-MA treated SGT cells. In YD-10B cells, the HCA-induced LC3B level was also suppressed by the addition of 3-MA (Fig. 7B). However, unlike SGT cells, the HCA-induced PARP cleavage in YD-10B cells was inhibited by 3-MA treatment, indicating that autophagy induces apoptosis in these cells. A siRNA experiment against Atg5 was performed to inhibit the formation of the autophagosome. Fig. 7A and B show that Atg5 knock-down increased the levels of cleaved PARP in SGT cells and decreased the level of cleaved PARP in YD-10B cells (Fig. 7C). Taken together, these results suggest that autophagy might contribute to apoptosis in head and neck cancer cells treated with HCA. The inhibition of apoptosis by autophagy inhibitors was confirmed by visualizing the green fluorescence in YD-10B cells (Fig. 7D). We then examined whether the overexpression of LC3B affects HCA-mediated apoptosis. For this experiment, the cells were transfected with LC3B expressing vectors and the correlation between autophagy and apoptosis was assessed by detecting the levels of cleaved PARP. Notably, the overexpression of LC3B decreased the levels of cleaved PARP in SGT cells compared with the HCA-treated control. However, the overexpression of LC3B increased the levels of cleaved PARP in YD-10B cells (Fig. 7E). These results strongly suggest that autophagy may actively contribute to the HCA-induced apoptosis in YD-10B cells and negatively regulates HCA-induced apoptosis in SGT cells.

\section{Discussion}

In the present study, we showed that the cinnamaldehyde derivatives, HCA and BCA, have potent anti-proliferative and cytotoxic activity against p53-wild-type (SGT) and p53-mutant (YD-10B) head and neck cancer cells. We also observed that the HCA- and BCA-treated cells have apoptotic morphologies, reductions in the pro-forms of caspase-3, -7 and -9 , and increased levels of PARP cleavage, implying the induction of apoptosis via the mitochondrial caspase-dependent signaling pathway. Our findings are consistent with other reports showing that HCA and BCA trigger apoptosis in MDA-MB-231 breast cancer cells and SW620 colon cancer cells by activating caspase-3 and PARP while suppressing the expression of antiapoptotic proteins such as Bcl-XL and Bcl-2 (4).
Genetic defects and mutations in the tumor suppressor gene p53 have been observed in various types of human malignancies (22). Many studies have demonstrated that p53 induces the expression of genes involved in cell cycle control, DNA repair, and apoptosis in response to DNA damage $(22,23)$. We observed that HCA treated SGT cells (p53-wild-type) exhibited p53 activation. Consistent with the role of p53 as a cell cycle regulator, we also observed the increased expression of p21 in SGT cells. Notably, p53 expression was not observed following HCA treatment in p53-mutant YD-10B cells; instead, the accumulation of p21 was observed. Although the cellular stress-induced upregulation of p53 has been used as an indicator of p53-dependent apoptosis in cancer cells $(23,24)$, our data demonstrated that HCA and BCA exhibit similar cytotoxic effects in p53-wild-type SGT cells and p53-mutant YD-10B cells which suggests a p53-independent cytotoxic mechanism.

Bcl-2 family members and mitochondria are important targets of p53 (25). Generally, the apoptotic pathway is activated by changes in the balance between anti- and proapoptotic members of the Bcl-2 family. Upon activation, Bak and Bax, the pro-apoptotic members of the Bcl-2 family, oligomerize and permeabilize the outer mitochondrial membrane, resulting in the release of cytochrome $c$, the activation of caspase-9 and subsequent PARP cleavage. Our data showed that HCA mediates apoptosis via a pathway involving the modulation of Bak1 and Bcl-2 as well as the initiator caspase proteins of the intrinsic pathway, caspase-3, -7 and -9. HCA induced the expression of Bak1 and decreased the expression of Bcl-2 in SGT and YD-10B cells. The levels of Bcl-XL and Bid remained unchanged. Previous studies have shown that phospho-p53 functionally re-organizes the Bak/Bcl-XL complex and activates $\operatorname{Bak}(20,24,25)$. We could not find any different activation patterns of Bcl-2 family members in either the p53-wild-type (SGT) or p53-mutant type (YD-10B) cells. Additionally, HCA showed growth-inhibitory activity as well as apoptosis induction in both cell lines, suggesting HCA is a potent anticancer agent that induces mitochondria-mediated apoptosis regardless of the p53 status.

Autophagy has been shown to engage in a complex interplay with apoptosis. It has been suggested that the autophagic response observed in cells treated with diverse cytotoxic agents is involved in protecting cells from apoptosis. Alternatively, autophagy may be associated with a mechanism contributing to apoptosis (26-28). Despite these studies, the relationship between autophagy and apoptosis in head and neck cancer cells remains poorly understood.

We found that HCA-induced autophagy leads to the regulation of the apoptotic pathway. 3-MA, a specific inhibitor of the early autophagic process, strongly induced the activation of PARP in HCA-treated SGT cells. However, the suppression of LC3B by 3-MA blocked the HCA-induced PARP activation in YD-10B cells. In addition, HCA-induced apoptosis was inhibited by the overexpression of LC3B in SGT cells, while promoted in YD-10B cells. These findings suggest that the HCA-induced autophagy is associated with the mechanism contributing to apoptosis in SGT and YD-10B cells.

In conclusion, we demonstrated for the first time that HCA induces autophagy and apoptosis in head and neck cancer cells. Both HCA and BCA can trigger the intracellular 
cell death pathway in a p53-independent manner. However, HCA-induced apoptosis was alternatively regulated by LC3Bmediated autophagy, i) apoptosis in HCA-treated YD-10B cells was blocked by the addition of 3-MA, ii) HCA-induced apoptosis in SGT cells was increased by 3-MA. Therefore, our results suggest HCA as a potential therapeutic candidate for human head and neck cancer.

\section{Acknowledgements}

The present study was supported by the National Research Foundation of Korea (NRF) grant funded by the Korea government MSIP (no. 2008-0062283).

\section{References}

1. Jeong HW, Han DC, Son KH, Han MY, Lim JS, Ha JH, Lee CW, Kim HM, Kim HC and Kwon BM: Antitumor effect of the cinnamaldehyde derivative CB403 through the arrest of cell cycle progression in the G2/M phase. Biochem Pharmacol 65: 1343-1350, 2003.

2. Chao LK, Hua KF, Hsu HY, Cheng SS, Lin IF, Chen CJ, Chen ST and Chang ST: Cinnamaldehyde inhibits pro-inflammatory cytokines secretion from monocytes/macrophages through suppression of intracellular signaling. Food Chem Toxicol 46: 220-231, 2008

3. Kwon BM, Lee SH, Cho YK, Bok SH, So SH, Youn MR and Chang SI: Synthesis and biological activity of cinnamaldehydes as angiogenesis inhibitors. Bioorg Med Chem Lett 7: 2473-2476, 1997.

4. Han DC, Lee MY, Shin KD, Jeon SB, Kim JM, Son KH, Kim HC, Kim HM and Kwon BM: 2'-benzoyloxycinnamaldehyde induces apoptosis in human carcinoma via reactive oxygen species. J Biol Chem 279: 6911-6920, 2004.

5. Lee CW, Hong DH, Han SB, Park SH, Kim HK, Kwon BM and Kim HM: Inhibition of human tumor growth by 2'-hydroxyand 2'-benzoyloxycinnamaldehydes. Planta Med 65: 263-266, 1999.

6. Koh WS, Yoon SY, Kwon BM, Jeong TC, Nam KS and Han MY: Cinnamaldehyde inhibits lymphocyte proliferation and modulates T-cell differentiation. Int J Immunopharmacol 20: 643-660, 1998

7. Ka H, Park HJ, Jung HJ, Choi JW, Cho KS, Ha J and Lee KT: Cinnamaldehyde induces apoptosis by ROS-mediated mitochondrial permeability transition in human promyelocytic leukemia HL-60 cells. Cancer Lett 196: 143-152, 2003

8. Lee CW, Lee SH, Lee JW, Ban JO, Lee SY, Yoo HS, Jung JK, Moon DC, Oh KW and Hong JT: 2-hydroxycinnamaldehyde inhibits SW620 colon cancer cell growth through AP-1 inactivation. J Pharmacol Sci 104: 19-28, 2007.

9. Hong SH, Kim J, Kim JM, Lee SY, Shin DS, Son KH, Han DC, Sung YK and Kwon BM: Apoptosis induction of 2'-hydroxycinnamaldehyde as a proteasome inhibitor is associated with ER stress and mitochondrial perturbation in cancer cells. Biochem Pharmacol 74: 557-565, 2007.

10. Kim SA, Sung YK, Kwon BM, Yoon JH, Lee H, Ahn SG and Hong SH: 2'-Hydroxycinnamaldehyde shows antitumor activity against oral cancer in vitro and in vivo in a rat tumor model. Anticancer Res 30: 489-494, 2010.
11. Kang HS, Ock J, Lee HJ, Lee YJ, Kwon BM and Hong SH: Early growth response protein 1 upregulation and nuclear translocation by 2'-benzoyloxycinnamaldehyde induces prostate cancer cell death. Cancer Lett 329: 217-227, 2013.

12. Ock J, Lee HA, Ismail IA, Lee HJ, Kwon BM, Suk K, Lee WH and Hong SH: Differential antiproliferation effect of 2'-benzoyloxycinnamaldehyde in K-ras-transformed cells via downregulation of thiol antioxidants. Cancer Sci 102: 212-218, 2011.

13. Kwon JY, Hong SH, Park SD, Ahn SG, Yoon JH, Kwon BM and Kim SA: 2'-Benzoyloxycinnamaldehyde inhibits nitric oxide production in lipopolysaccharide-stimulated RAW 264.7 cells via regulation of AP-1 pathway. Eur J Pharmacol 696: 179-186, 2012.

14. Young MR, Levingston $\mathrm{C}$ and Johnson SD: Cytokine and adipokine levels in patients with premalignant oral lesions or in patients with oral cancer who did or did not receive $1 \alpha, 25$ dihydroxyvitamin D3 treatment upon cancer diagnosis. Cancers (Basel) 7: 1109-1124, 2015.

15. Moon SM, Ahn MY, Kwon SM, Kim SA, Ahn SG and Yoon JH: Homeobox C5 expression is associated with the progression of 4-nitroquinoline 1-oxide-induced rat tongue carcinogenesis. J Oral Pathol Med 41: 470-476, 2012.

16. William WN Jr and El-Naggar AK: A novel target for oral cancer chemoprevention? Notch quite, yet.... Cancer Prev Res (Phila) 8: 262-265, 2015.

17. Lee EJ, Kim J, Lee SA, Kim EJ, Chun YC, Ryu MH and Yook JI: Characterization of newly established oral cancer cell lines derived from six squamous cell carcinoma and two mucoepidermoid carcinoma cells. Exp Mol Med 37: 379-390, 2005.

18. Kim SA, Kim YC, Kim SW, Lee SH, Min JJ, Ahn SG and Yoon JH: Antitumor activity of novel indirubin derivatives in rat tumor model. Clin Cancer Res 13: 253-259, 2007.

19. Zhang L, Yu J, Park BH, Kinzler KW and Vogelstein B: Role of BAX in the apoptotic response to anticancer agents. Science 290: 989-992, 2000

20. Pietsch EC, Perchiniak E, Canutescu AA, Wang G, Dunbrack RL and Murphy ME: Oligomerization of BAK by p53 utilizes conserved residues of the p53 DNA binding domain. J Biol Chem 283: 21294-21304, 2008.

21. Oda E, Ohki R, Murasawa H, Nemoto J, Shibue T, Yamashita T, Tokino T, Taniguchi T and Tanaka N: Noxa, a BH3-only member of the Bcl-2 family and candidate mediator of p53-induced apoptosis. Science 288: 1053-1058, 2000.

22. Soussi T and Lozano G: p53 mutation heterogeneity in cancer. Biochem Biophys Res Commun 331: 834-842, 2005.

23. Vogelstein B, Lane D and Levine AJ: Surfing the p53 network. Nature 408: 307-310, 2000.

24. Oren M: Decision making by p53: Life, death and cancer. Cell Death Differ 10: 431-442, 2003.

25. Vaseva AV and Moll UM: The mitochondrial p53 pathway. Biochim Biophys Acta 1787: 414-420, 2009.

26. Levy JM and Thorburn A: Targeting autophagy during cancer therapy to improve clinical outcomes. Pharmacol Ther 131: 130-141, 2011.

27. Chen S, Rehman SK, Zhang W, Wen A, Yao L and Zhang J: Autophagy is a therapeutic target in anticancer drug resistance. Biochim Biophys Acta 1806: 220-229, 2010.

28. Choi KS: Autophagy and cancer. Exp Mol Med 44: 109-120, 2012. 